\title{
A posture prediction method for ergonomic assessment of user-product interactions while grasping using musculoskeletal human models
}

\author{
Carla Hartmann ${ }^{1 *}$, Alexander Wolf ${ }^{1}$, Jörg Miehling ${ }^{1}$, Sandro Wartzack ${ }^{1}$ \\ ${ }^{1}$ Engineering Design, Friedrich-Alexander-Universität Erlangen-Nürnberg \\ * Corresponding author: \\ Carla Hartmann \\ Engineering Design \\ Martensstraße 9 \\ 91058 Erlangen \\ Phone: +49 (0)911/85-96618 \\ Mail: hartmann@mfk.fau.de
}

\begin{abstract}
Using digital human models (DHMs) as the virtual representation of the human body enables the integration of ergonomic assessments in the product design process. Especially the use of DHMs in the early phases of product design can be decisive for competitive factors such as ergonomics or usability. Therefore, we present a method for automatic posture generation for grasping tasks using a musculoskeletal human model. This method enables ergonomic analyses without ergonomic knowledge of the product designer or subject tests. We implemented the presented method for a cylinder grasping scenario using the open source software OpenSim. Subsequently, we evaluated the implementation by means of a parameter study.
\end{abstract}

\section{Keywords}

Posture prediction, musculoskeletal simulation, digital human model 


\section{Motivation}

Decades ago, the scientific field of ergonomics already found its way into the design of technical systems. It shows how technical systems need to be designed for being used efficiently by humans without damage or fatigue. It occurred that ergonomics is also of great importance in product design and can be decisive for the acceptance and thus the financial success of a product. For this reason, the integration of the potential users' needs in the product design process is advantageous [1]. One way to achieve this is the usage of digital human models (DHMs) as the virtual representation of the human. These are part of software systems and can model not only the geometry but also other human characteristics and abilities [2]. Thus, DHMs can for example be used to simulate humans of variant size and help designing products usable for a large variety of people. In general it appears that the integration of DHMs in the product design process saves a lot of time and money compared to traditional methods for design testing [3].

\section{State of the art}

\subsection{The hand: Anatomical basics and its use for grasping objects}

With the development of the upright gait in humans' evolution, the locomotion function of the front limbs was eliminated and their use changed fundamentally. Due to the mobility of the shoulder joints and the hand's large range of motion, the hand developed into the most important organ for grasping and feeling objects. In cooperation with the brain, the hand was of great importance to humans' evolution and can be termed as the humans' "cultural organ". The anatomy of the forearm and the hand is of great significance for the hand's multifunctionality. As depicted in Figure $1 \mathrm{a}$ ), the forearm is composed of the radius and the ulna. The hand itself is divided into the carpus, the metacarpus and the fingers. The muscular system of the forearm and the short muscles in the metacarpus realize the finger movements. As a result, the fingers themselves have no musculature and therefore a slender structure. This slim structure and the oppositional position of the thumb are essential for the grasping and sensing function of the hand [4].

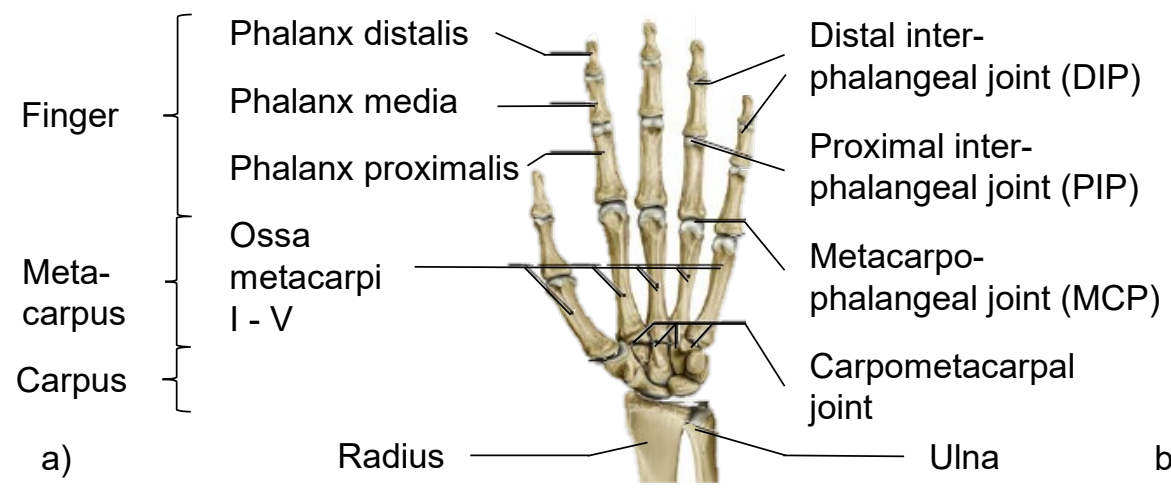

b)

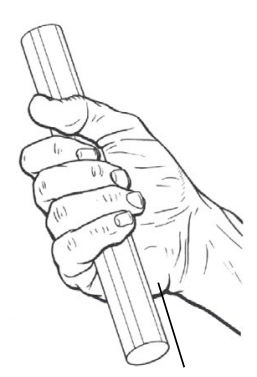

Hypothenar muscle

Figure 1: Section a) shows the hand's bone structure [5], while in b) a palm grip using the thumb is depicted [6].

Because of its anatomy, the hand is able to perform a variety of grasping tasks divided into different categories. If the palm is used for a grasping task, the task is termed as "palm grip". In dependence on the geometry of the grasped object, a palm grip can be performed with or without the thumb. A palm grip with the thumb presents the safer grasp form and is mostly used for grasping comparatively large and heavy objects. Figure $1 \mathrm{~b}$ ) depicts a palm grip using the thumb while grasping a cylindrical object. Using this grasping task, the cylinder's longitudinal axis coincides with the hand gutter. This gutter is located on the palm and runs diagonally from the hypothenar muscle to the head of the second metacarpale [6]. 


\subsection{Digital human models in product design}

Usability can be one of the most important factors for the acceptance and the financial success of a product. Consequently, focusing on user-centered methods in product design processes has a positive influence on product's success [7]. This can be achieved, for example, by using DHM. One important sub-category of all currently used DHMs is represented by the anthropometric DHMs. These DHMs are part of common computer-aided design (CAD) systems and are typically used for ergonomic visualizations such as reach analyses. However, DHMs integrated in common CAD programs require manual adjustments to model's posture based on the object's geometry. These adjustments demand basic ergonomic understanding. For conducting biomechanical analyses for the prediction of forces acting in the human body, this type of DHMS can only be used to a limited extent. For such dynamic analyses, musculoskeletal DHMs are preferred. In these models, structures such as bones, tendons and muscles are modelled with particular anatomical accuracy. Therefore, musculoskeletal DHMs are particularly suitable for analyses of muscle forces and joint reactions occurring during movements [2]. However, most DHMs, including musculoskeletal DHMs, have limitations in motion generation. By using motion capture techniques, movements of subjects are recorded while using product prototypes and transmitted to musculoskeletal DHMs. Afterwards it is possible to perform biomechanical analyses for these movements [8].

Despite the great advantage of using DHMs in the product design process, the DHMs lack a sufficient possibility of modelling user-product interactions to integrate them better in product design processes. Therefore, Wolf et al. [9] present a method for interaction modelling based on the concept of affordances. The term affordances was introduces by the psychologist James J. Gibson and describes interaction possibilities provided or afforded by a product or environment towards an actor [10]. A simple example of an affordance is that a doorknob affords the user to turn it. Similar to this example, the concept of affordances can be used to describe a variety of complex relationships between systems. As a consequence, the concept of affordance is not only used in psychology, but has also gained importance in product design [11]. Based on the affordance concept, Wolf et al. [9] present a promising approach for modelling user-product interactions with DHM for virtual ergonomics assessments.

\subsection{Musculoskeletal simulation}

For conducting biomechanical simulations with a musculoskeletal DHM, motions and reaction forces have to be recorded from a subject and transferred to the DHM. For recording movements, techniques such as motion capturing are normally used [12]. Here, the entire human movement is recorded with the help of a limited number of markers placed on the real object. The position or orientation of the markers is then transferred to the digital model. For this purpose, the DHM must be equipped with markers that are placed at the same positions in the model as on the human who performs the movement. For transferring the motion on the $\mathrm{DHM}$, inverse kinematics $(\mathrm{IK})$ is used. Based on the last-squares problem the IK minimizes the difference between the location of DHM's virtual markers and the measured marker location. As a consequence, for each time step all joint angles of the DHM are adjusted that the sum of all marker differences is minimal [13].

Based on the movement determined by the IK and external forces recorded during the subject's motion, a dynamic simulation can be conducted. Inverse dynamics enables the prediction of the generalized forces at each joint, which produce the movement. Furthermore, static optimization (SO) calculates the forces and activations of all muscles, which lead to the observed motion.

Beside the common approach of motion simulation based on subject tests, there are already some procedures for motion prediction without prior experimental collection of specific motion data. This saves the time-consuming, expensive and possibly dangerous execution and 
recording of motion sequences with subjects [14]. Farahani et al. [8] present an approach by using constrained optimization with limitations of the DHM's locomotor apparatus for a predictive motion generation. This approach was implemented for conducting a squat vertical jump with maximal height. Caused by the high computation effort and the application specific optimization, this approach is however only limited applicable in product development. For purposes of product design processes, a more general movement prediction depending on user and product characteristics is necessary. Therefore, Wolf et al. [15] present a promising approach for predicting DHMs' movement by the combination of a motion database and a datadriven method with a constrained optimization algorithm. Based on the database, consisting different movements of the same interaction, the regression model predicts a movement strategy. This is expressed in from of time-curves for positions and/or joint angles. Subsequently, the whole body movement is predicted based on these time-curves. In a pilot study, this approach was implemented for lifting of an object in the sagittal plane. In general, this seems to be a promising concept for predicting specific physiological movements of DHMs based on a motion database created by experimental data from subject tests.

\section{Research gap and goal}

Despite the advantages of using DHMs in early product design processes, the used techniques have some limitations. As described above, DHMs integrated in common CAD applications can be applied in the early product design processes. However, these models have several restrictions, such as the ergonomic understanding from the designer for manual adjustments or the limited possibility of conducting biomechanical analyses. Musculoskeletal DHMs, which are usually used for conducting biomechanical analyses, are mostly applied in the later stages of the product design process. This is due to the fact that usually experimentally measured data from subjects are required.

Based on the advantages of considering ergonomics in design processes and the presented limitation of using DHMs especially in the early phases of the product design process, the following research question can be derived: How can musculoskeletal DHMs be better integrated into the early product design process for conducting biomechanical analyses and first ergonomic assessments?

Concerning this question, we present a method for posture prediction with a musculoskeletal DHM. Using this method no manual adjustments to the model's posture based on the object's geometry and thus no ergonomic understanding from the designer are necessary. The method depicts a possibility for an easily conducted posture prediction of the musculoskeletal DHM while interacting with an object. This posture prediction enables ergonomic assessments without any experimentally measured data from subject tests. Based on the taxonomy for user-product interaction, set up by Wolf et al. [9], we defined the method for the affordance "grasping" an object.

\section{Method}

We present a general method for automatically predicting the posture of a musculoskeletal DHM while conducting the affordance "grasping" an object. Afterwards, we explain the implementation of this method for the affordance "grasping a cylinder" with a palm grip. This grasped cylinder could for example represent a gear lever of a machine. For the implementation we used the open-source software OpenSim and its interface to Matlab. [12]

\subsection{General method for posture prediction}

As depicted in Figure 2, the base for generating the DHM's posture is the information about position and geometry of the object. In addition, we use a musculoskeletal DHM. Based on the 
object's geometry, constraints in form of virtual markers, used for conducting the IK, are added to the DHM' hand. Using the virtual markers enables a flexible adaption on the objects geometry and therefore a wide range of grasping tasks can be generated. For these markers a linear movement from the start position in the human's upright posture to the end position is generated. Therefore, the hand markers' position is calculated for each time step of the movement. Based on these constraints for the markers, we generated a motion of the DHM by using IK. Afterwards, all joint angles of the DHM in the last time step of the motion are used to define the final posture. Subsequently, a dynamic simulation for analysing muscle forces and joint reactions can be performed based on this posture. For these analyses, external forces can be introduced simplified via a single force vector. This enables the simulation of interactions between the user and an object.

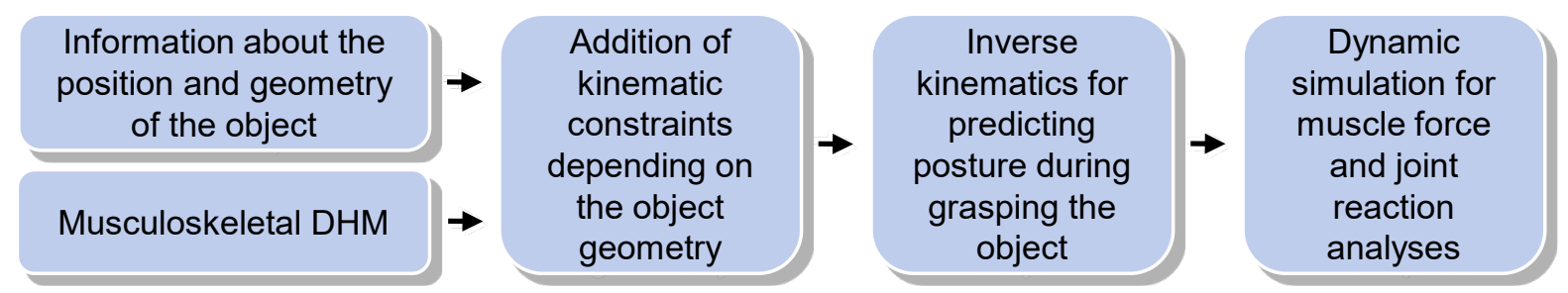

Figure 2: General method for grasping an object with a musculoskeletal DHM.

\subsection{Implementation of grasping a cylinder}

For evaluating the presented general method we implemented it for the affordance "grasping a cylinder" in the handling area with a palm grip by using the thumb (Figure $1 \mathrm{~b}$ )). The prediction of the final posture depends on the grasping object's geometry and its position related to the user. We use the start and end point of the cylinder's longitudinal axis as well as the cylinder's radius as the base for the posture prediction. These required data are illustrated in Figure $3 \mathrm{a}$ ). For conducting a palm grip by using the thumb, the cylinder's longitudinal axis has to coincide with the hand gutter. As described in 2.1, the hand gutter runs diagonally from the hypothenar muscle to the head of the second metacarpale. For this reason, two markers were placed on the DHM's palm as depicted in Figure $3 \mathrm{~b}$ ). The first one was placed on the head of the second metacarpale, the other on the beginning of the hypothenar muscle. These two markers must coincide with the cylinder's longitudinal axis to define the final position of the hand.

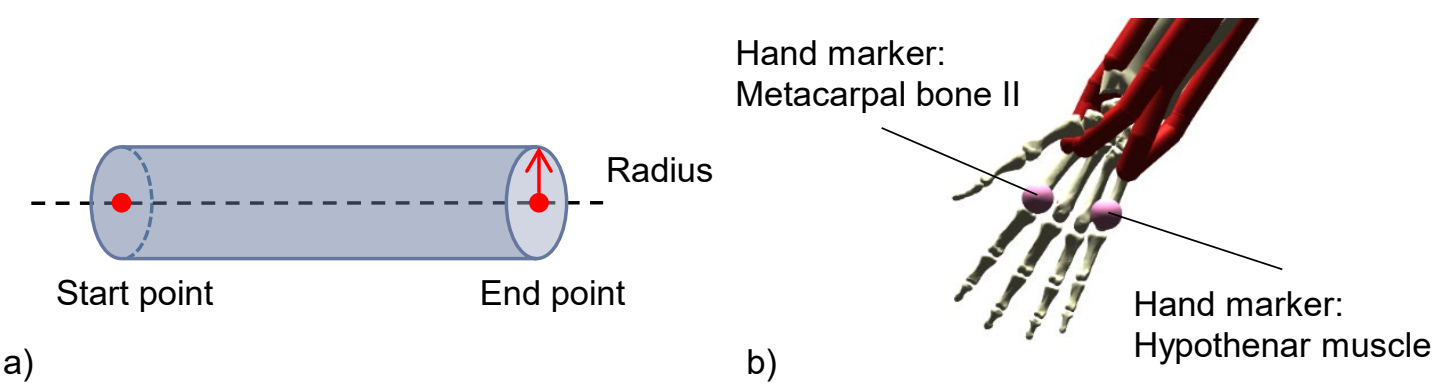

Figure 3: Section a) depicts the information regarding the cylinder geometry, which is the basis for the posture prediction. Both hand markers, placed on the DHM's palm, are shown in Section b).

However, if the hand markers are placed directly on the palm, the hand is inside the cylinder and not on its surface. This case is depicted on the left side of Figure $4 \mathrm{a}$ ). Consequently, both hand markers must be adjusted. For this, the distance between hand markers and palm is always equivalent to the cylinder's radius. This adjustment was automated via the Mablab interface based on the cylinder's geometry information. As a consequence of this adjustment, the palm will be placed on the cylinder's surface as depicted on the right side of Figure $4 \mathrm{a}$ ). 
To enable an automatic adjustment of the finger joint coordinates to the cylinder's radius we generated a database including values for all finger joint angles. We manually adjusted the finger joints for three radii, as depicted in Figure $4 \mathrm{~b}$ ), and used the resulting joint angles as database. Based on this data, the finger joints angles can be linearly adjusted for radii ranging from $15 \mathrm{~mm}$ to $35 \mathrm{~mm}$. Thus, the palm grip while using the thumb can be adapted automatically to the cylinder's geometry.

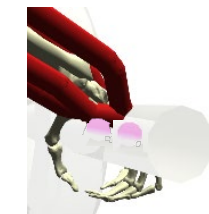

a)

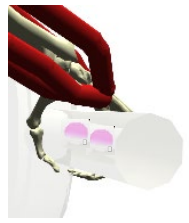

b)

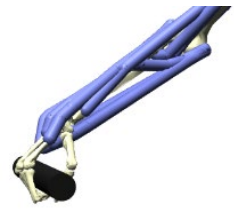

$\mathrm{r}=15 \mathrm{~mm}$

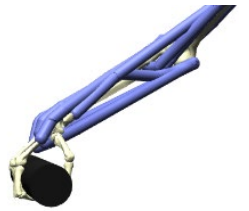

$\mathrm{r}=25 \mathrm{~mm}$

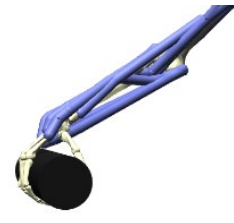

$\mathrm{r}=35 \mathrm{~mm}$

Figure 4: Section a) shows the necessary adjustment of the hand markers based on the cylinder's radius. The adaptation of the finger joint angles to three cylinders with different radii $(r)$ is depicted in section $b$ ).

\subsubsection{Posture generation via inverse kinematics}

For generating the final posture of the musculoskeletal DHM grasping the cylinder, we use the IK. We defined the start position for the IK as the neutral, upright posture in which the hand is located beside the body. In the end position, the palm is on the cylinder's surface and the angles of the finger joints are adapted to the cylinder's radius. To make the IK more robust we use several iteration steps for generating the final posture. Consequently, for reaching the final posture, we define a motion sequence. This motion sequence lasts five seconds (250 time steps) and is depicted in Figure 5.

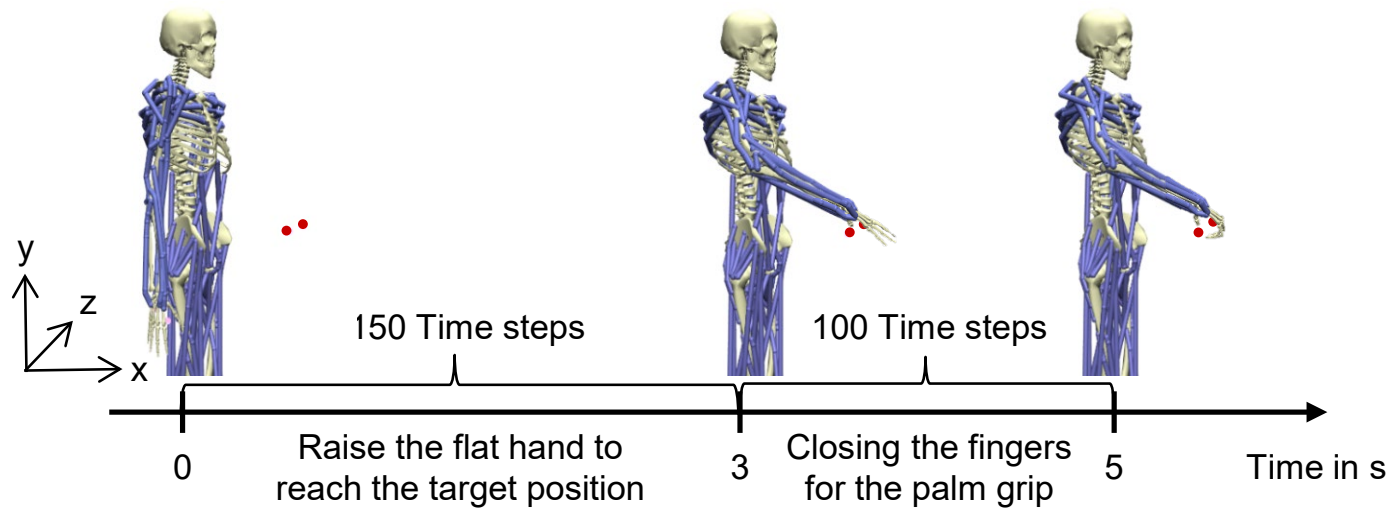

Figure 5: Motion sequence of grasping a cylinder with a palm grip depending on cylinder parameters. The red points depict the start and end of the cylinders longitudinal axis.

For the first part of this sequence, we define a straight motion line for the two hand markers from their start to their end position on the cylinder's longitudinal axis. Based on this constraint for the hand markers, the DHM raises the flat hand to the end position of the hand markers and thereby positions its flat hand on the cylinder's surface. This first part of the motion sequence for generating the final posture takes three seconds, divided into 150 time steps. After positioning the palm on the cylinder's surface, the fingers close around the cylinder. As shown in Figure 5, the adaption of the finger joint angles represents the last two seconds of the motion sequence, divided into 100 time steps. Consequently, the final posture for grasping the cylinder is defined by all joint angles of the body in the end position of the motion sequence shown in Figure 5. 


\subsubsection{Identification of muscular forces via static optimization}

Based on the posture generated by using the IK, dynamic analysis can be performed with the SO. This predicts for each muscle the force as well as the activation responsible for the posture. Therefore, external forces acting on the DHM must be defined previously. By analyzing only the posture's effect on the muscular system, only the ground reaction forces represent the external forces. These kind of forces are normally part of the experimentally measured database. Because of our method's independence of subject based experimental data, residual actuators are used to stabilize the DHM on the ground and simulate the ground reaction forces. For the stabilization of the DHM on the ground, these actuators are allocated to the DHM's right and left calcaneus. The actuators' force limits are set to $100 \mathrm{~N}$ for the $\mathrm{x}$ - and $\mathrm{z}$-direction and to $500 \mathrm{~N}$ for the y-direction. The maximum for the actuator moments is set to $100 \mathrm{Nm}$ for all directions.

For a better prediction of the influence of user-product interactions, additional external forces can be considered for the SO. For predicting the influence of pushing or pulling the cylinder, a force in negative or positive $\mathrm{x}$-direction can be applied. As a simplification we apply the external force for pushing or pulling to the third metacarpal bone. As shown in Figure $1 \mathrm{a}$ ), this bone is located in the middle of the metacarpus and for this reason it is suitable for simplifying the force application on the palm.

\subsubsection{Study design for the evaluation of the implementation}

For evaluating the described method and its implementation, we conducted a parameter study in order to predict the DHM's posture and the resulting muscular forces while grasping cylinders in different locations. We defined three different scenarios, which are depicted in Figure 6.

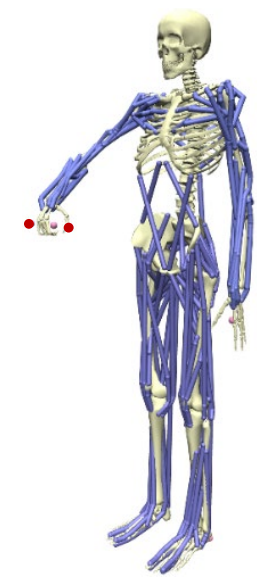

Variant 1

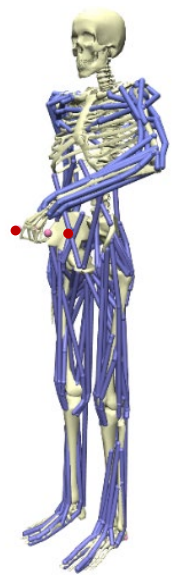

Variant 2

Scenario 1

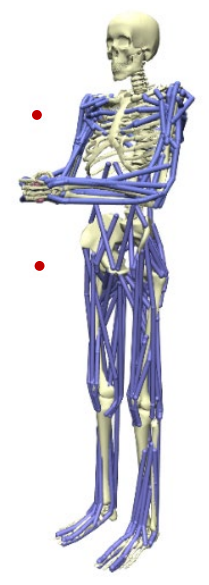

Scenario 2

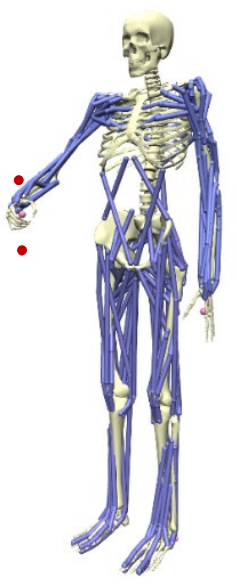

Scenario 3

Figure 6: Different possible scenarios for grasping a cylinder in the handling area. The red points depict the start and end of the cylinders longitudinal axis.

For all scenarios we used a cylinder with a radius of $25 \mathrm{~mm}$. In Scenario 1 we placed a horizontal cylinder at waist level in front of the DHM. For the Variant 1 of the first scenario, the cylinder is located directly in front of the right arm, for Variant 2 in front of the left arm. Consequently, we defined the right hand for grasping the cylinder in Variant 1 and the left hand in Variant 2. For the evaluation of grasping tasks with both hands, we defined Scenario 2. In this scenario, the DHM is grasping a vertical cylinder, located in the middle of the body and in front of the DHM. For this grasping task the hand markers' final position is identical for both hands. Because of this, both hands interlock. Using Scenario 3, we evaluate the possibility of 
posture prediction for grasping cylinders which are neither horizontal nor vertical to the ground. For this, the coordinates of the cylinder's axis start and end point differ in all directions. Accordingly, for Scenario 3 we placed a slanted cylinder in the handling area of the DHM's right hand.

For evaluating our method regarding to the prediction of muscular forces we use different force conditions. First we define the normal state in which only the influence of the posture can be evaluated. Furthermore, force conditions for pulling and pushing the cylinder are determined. For these force conditions a pull or push on the cylinder with a total force of $20 \mathrm{~N}$ or $50 \mathrm{~N}$ is defined.

\section{Results and discussion}

\subsection{Kinematic analysis}

The evaluation study was conducted with a full body DHM with a mass of $73.3 \mathrm{~kg}$ and 150 muscles. For evaluating the quality of the posture prediction for the hand, we calculated the difference between the predefined end positions of the hand markers on the cylinder' longitudinal axis and their position in the predicted posture. An overview of the differences for all three scenarios is depicted in Figure 7.

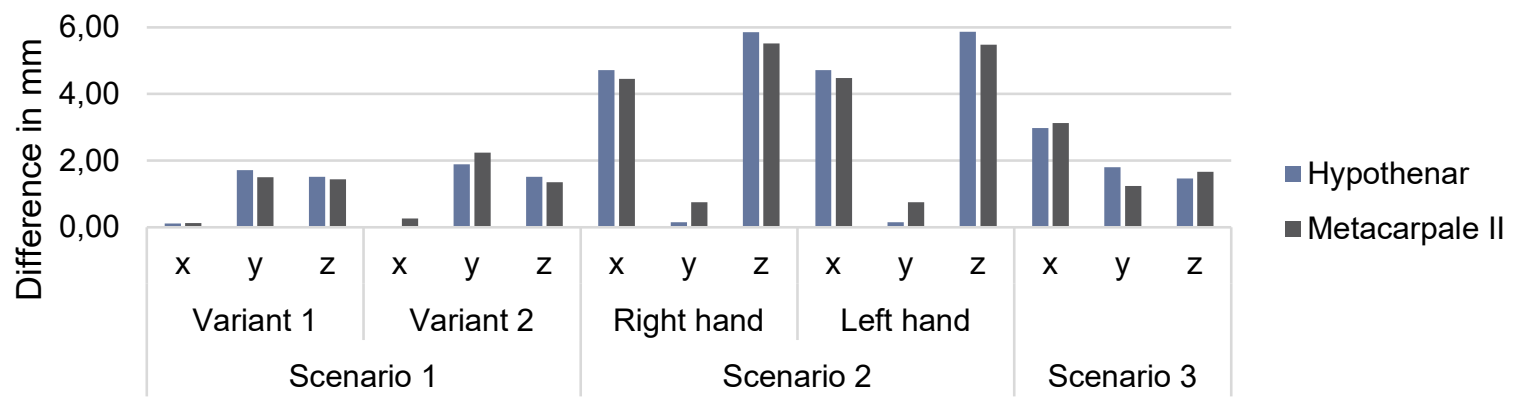

Figure 7: The difference between the predefined position of the hand markers and their position in the predicted posture.

It turns out that in all scenarios the hand maker did not exactly reach the predefined position. The smallest difference for both hand markers occurs in Scenario 1 (grasping a horizontal cylinder). For both variants of Scenario 1 the difference for the $x$-coordinates is less than $0.2 \mathrm{~mm}$. So, in the $\mathrm{x}$-direction the hand markers have reached the predefined position almost perfectly. In general, all of the differences for Scenario 1 are very low with values under $2.3 \mathrm{~mm}$. In contrast, the highest values are achieved in Scenario 2. The position of the hand markers deviates in the z-direction up to $5.9 \mathrm{~mm}$ from the predefined position. Furthermore, the differences in Scenario 2 are symmetrical for the right and the left hand. Because of the identical predefined position of both hand markers in the middle of the body, this indicates that the presented method predicts equal results for each side of the body. Grasping a cylinder, which is neither horizontally nor vertically to the ground in the handling area, is represented by Scenario 3. In this scenario, the differences of hand marker positions are between the values of Scenario 1 and 2.

\subsection{Dynamic analyses}

In addition to the evaluation of the predicted posture after the IK, the possibility for conducting biomechanical analyses based on this posture should be assessed. For this assessment, the SO for the predefined force conditions was conducted based on the postures of Scenario 1. As expected, different force conditions caused different muscle forces and 
activations. An overview of the muscle activation for all force conditions based on Scenario 1 Variant 1 is shown in Figure 8. The color of the individual muscles symbolizes their activation. While muscles with low activation are colored blue, the color changes to red with increasing activation. Figure 8 shows that the muscle activation without the influence of additional forces, i. e. only while holding the posture, was very low. A higher activation of individual muscles can only be observed by the application of external forces, in this case by pulling or pushing the cylinder. In addition, Figure 8 illustrates that the activation rises with increasing external forces. When an external force of $50 \mathrm{~N}$ is applied, the muscles show a significantly higher activation than by a force application of $20 \mathrm{~N}$. This increase in muscle activation by load increase was also observed by Bennett et al. [16] when performing EMG analyses for various pull and push techniques by maneuvering a loaded pallet jack.

Furthermore, the activated muscles for pulling or pushing were different. While pulling the cylinder, particular the flexor muscles of the forearm and on the calf indicates high activation. Contrary to this, pushing the cylinders caused a high activation of extensor muscles of the forearm and the shoulder as well as of muscles of the shin. These results are identical for Variant 2 of the first scenario.

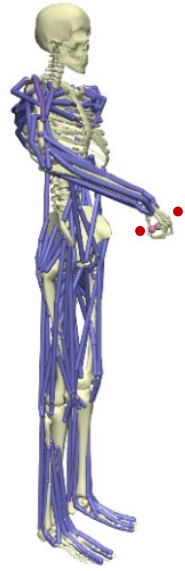

$0 \mathrm{~N}$

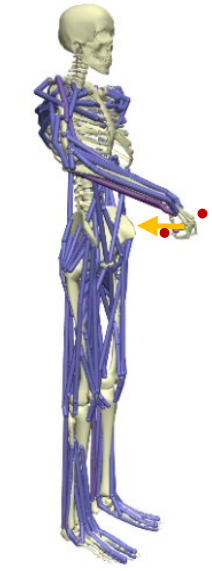

$20 \mathrm{~N}$

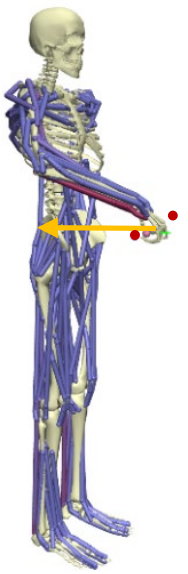

$50 \mathrm{~N}$

Pull

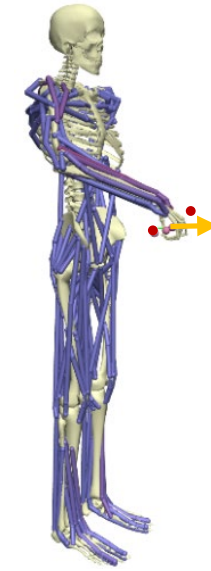

$20 \mathrm{~N}$

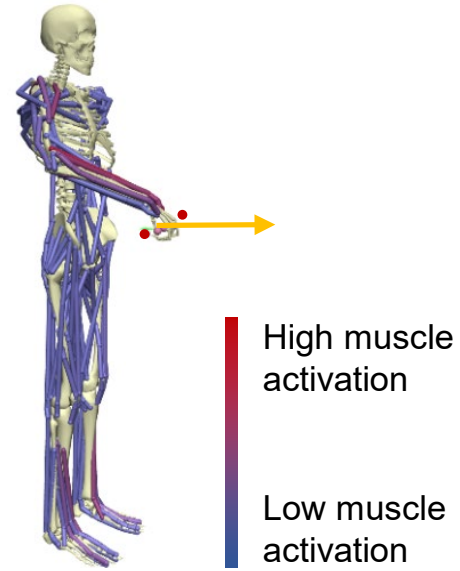

$50 \mathrm{~N}$

Push

Figure 8: Muscle activation based on the posture of Scenario 1 Variant 1 without force application as well as while pulling or pushing. The red points depict the start and end of the cylinders longitudinal axis.

\section{Conclusion and outlook}

We presented a method for automatic posture prediction for the affordance "grasping an object" in the handling area with a musculoskeletal DHM. This method is independent of any experimentally measured data from subject tests and ergonomic understanding from the designer. We implemented the presented method for the affordance "grasping a cylinder" with a palm grip using the thumb. By carrying out a parameter study we showed, that our method is suitable for automatic posture prediction for grasping a cylinder in different locations without experimentally measured subject data. In the generated posture the hand markers and therefore also the hand did not exactly reach the predefined position. Due to the small deviation of maximum $5.9 \mathrm{~mm}$, the method nevertheless seems to be suitable for a first ergonomic assessment of the posture resulting from a user-product interaction. In addition, we illustrated that the predicted posture can be used for conducting biomechanical analysis. Consequently, internal reactions of the human body can be estimated. In addition, interactions such as pulling or pushing the cylinder can be integrated, too.

In summary, the presented method and its implementation for the specific affordance "grasping a cylinder" with a palm grip using the thumb shows a promising approach for a better 
integration of musculoskeletal DHM in the early product design process. It depicts that a posture prediction only based on the objects geometry is possible. Nevertheless, for this pilot study we implemented only one specific affordance. Consequently, for evaluating the generalization of the presented method and a full applicability in the product design process a large number of further user-product interactions in form of affordances must be implemented. In the course of this, further parameter studies with a wider range of scenarios should be conducted. In addition, this approach only shows the possibility for easily predicting postures. For this purpose, the posture is generated based on a few constraints, whereby the model cannot react to external influences. Further research should be done for enabling the model's adaption to external kinematic and dynamic conditions. Thus, the posture could be predicted more realistically.

\section{Acknowledgements}

This research work is part of the FAU "Optimization-based design methodology in early phase of mechatronic product development" project (EFRE/OptMePro) and funded by the Bavarian program for the "Investment for growth and jobs" objective finance by the European Regional Development Fund (ERDF), 2014-2020. It is managed by the Bavarian Ministry of Economic Affairs and Media, Energy and Technology. The authors are responsible for the content of this publication.

\section{Literaturverzeichnis}

[1] Lindemann, Udo (Hrsg.): Handbuch Produktentwicklung. München: Hanser, 2016

[2] Bullinger-Hoffmann, Angelika C.; Mühlstedt, Jens: Homo Sapiens Digitalis - Virtuelle Ergonomie und digitale Menschmodelle. Berlin, Heidelberg: Springer Berlin Heidelberg, 2016

[3] Chaffin, Don B.: Improving digital human modelling for proactive ergonomics in design. In: Ergonomics 48 (2005), Nr. 5, S. 478-491

[4] Aumüller, Gerhard et al.: Duale Reihe Anatomie. Stuttgart: Georg Thieme Verlag, 2017

[5] Schünke, Michael et al.: PROMETHEUS Allgemeine Anatomie und Bewegungssystem. Stuttgart: Georg Thieme Verlag, 2018

[6] Kapandji, Adalbert I.; Koebke, Jürgen: Funktionelle Anatomie der Gelenke: Schematisierte und kommentierte Zeichnungen zur menschlichen Biomechanik; [obere Extremität, untere Extremität, Rumpf und Wirbelsäule]. 4. unveränd. Aufl.; einbd. Ausg. Stuttgart: Thieme, 2006

[7] Kujala, Sari et al.: The role of user involvement in requirements quality and project success. In: 13th IEEE International Conference on Requirements Engineering: Proceedings: Paris, France, 29 August - 2 September 2005. Los Alamitos, Calif.: IEEE Computer Society, 2005, S. 75-84

[8] Farahani, Saeed D. et al.: Optimization-based dynamic prediction of kinematic and kinetic patterns for a human vertical jump from a squatting position. In: Multibody System Dynamics 36 (2016), Nr. 1, S. 37-65

[9] Wolf, Alexander et al.: Approaching an ergonomic future: An affordance-based interaction concept for digital human models. In: Procedia CIRP 84 (2019), S. 520-525

[10] Gibson, James J.: The ecological approach to visual perception. Boston: Houghton Mifflin, 1979

[11] Maier, Jonathan R. A.; Fadel, Georges M.: Affordance based design: status and promise. In: Proceedings of IDRS (Nov. 2006), 10-11

[12] Delp, Scott L. et al.: OpenSim: Open-source software to create and analyze dynamic simulations of movement. In: IEEE transactions on bio-medical engineering 54 (2007), Nr. 11, S. 1940-1950

[13] Jackèl, Dietmar (Hrsg.); Neunreither, Stephan (Hrsg.); Wagner, Friedrich (Hrsg.): Methoden der Computeranimation. Berlin: Springer, 2006 (eXamen.press)

[14] Wolf, Alexander; Miehling, Jörg; Wartzack, Sandro: Challenges in interaction modelling with digital human models - A systematic literature review of interaction modelling approaches. In: Ergonomics (2020), S. 1-17

[15] Wolf, Alexander et al.: Towards Virtual Assessment of Human Factors: A Concept for Data Driven Prediction and Analysis of Physical User-product Interactions. In: Proceedings of the Design Society: International Conference on Engineering Design 1 (2019), Nr. 1, S. 4029-4038

[16] Bennett, A. I.; Todd, Andrew. I.; Desai, S. D.: Pushing and pulling, technique and load effects: an electromyographical study. In: Work (Reading, Mass.) 38 (2011), Nr. 3, S. 291-299 